\title{
The Overall Design of Digital Medical System under the Network Environment
}

\author{
Yuping Zhou ${ }^{1}$, Yicheng Zhou ${ }^{2}$, and Min $\mathrm{Yu}^{3, *}$ \\ ${ }^{1}$ The Collage of Information Science and Technology, Hainan Normal University, \\ Haikou, China \\ zypnew@qq. com \\ ${ }^{2}$ First Affiliated Hospital of Harbin Medical University, Harbin, China \\ yichengzhou2011@163.com \\ ${ }^{3}$ Academic Affairs Office, Hainan Normal University, Haikou, China \\ 704222535 @qq. com
}

\begin{abstract}
The wireless sensing network technology, communication technology, password technology, etc. were adopted to carry out the overall design to the digital medical system under the network environment, in order to realize the functions of the remote medical diagnosis, health care, locating and tracking, emergency calls and first aid. In this paper, the design method of the community and personal digital medical system was expounded and the overall design scheme of the digital medical system was provided, including the function module design such as the user terminal system, safety call system, electronic medical records system, hospital information management system, diagnosis expert system and emergency center control system.
\end{abstract}

Keywords: digital medical treatment, network environment, system design.

\section{Introduction}

With the rapid development of network technology and the continuous improvement of people's living standard, people have a higher request to the life quality; therefore, people have a higher request to the daily health care service. The traditional medical model of the diagnosis and treatment such as face to face between doctors and patients cannot meet the requirements of people. People's demand for health care is growing rapidly and the emergency medical treatment is required higher and higher.

Mobile digital hospital mainly involved in a number of sub-systems such as the hospital wireless clinical information systems, intelligent expert clinics, health tracking service system, remote medical monitoring system, community health telematics system, etc. It can create an all-round, new medical services platform for patients, in order to promote the establishment of new hospital service model. With the development of digital communications technology, the digital medical in the network environment will give people more and more help and services. Mobile digital hospital will become more and more common.

\footnotetext{
* Corresponding author.
} 
Wireless sensor network is the one that is organized by the sensor nodes in the way of wireless communication, in particular application environment. The sensor nodes were mainly used to collect data, and then, the collected data were sent to the network via a wireless sensor network, and finally received by the particular application system. Wireless sensor network is a new mode of information access and information processing.

It is very important to apply wireless sensor networks in the community and personal digital medical and to study out the digital medical system for the remote medical treatment, remote diagnosis, remote medical care, real-time nursing and emergency medical treatment. The system has advantages of the high reliability, high safety, high efficiency and humanization, etc. In this paper, the design of digital medical system under the network environment was introduced, including the system structure, system hardware platform, system software platform and its main function modules.

\section{$2 \quad$ Study Background}

A complete digital medical system should provide the personalized, digital medical service and health management platform for community residents. The platform system should include services such as the prevention, diagnosis, health care, medical care, first aid and interactive, etc. Therefore, it is necessary to comprehensively utilize the network technology, communication technology, control technology, and some medical equipment terminals to provide the medical service, health care service, medical care services, emergency services and health services for the residents.

Through the digital medical service system, community residents will get fast and convenient medical services. Residents can get SMS health information remind, remote medical diagnosis, remote health care, emergency treatment, expert interaction, online consultation and other services through fixed network or wireless network technology. In 1906, Wilhelm Einthoven, the inventor of the electrocardiogram, successfully carried out the remote consultation experiment through telephone lines, and become the first of the telemedicine development. In 1967, a radiologist in the United States established the interactive telemedicine system, which was the first to realize the doctor-patient interaction of remote medical system [1]. In 2006, 306th hospital of the people's liberation army carried out the wireless clinical medical to patients through the hospital local area network, and the wireless PDA was applied to query and monitor for patients sick signs, have a prescription, issue orders, make the mobile medical successfully by using the local area network, which greatly improved the efficiency of the doctor's work [2]. In 2012, Huaxiang and others developed "hospital information management system", whose main function was to realize the computerized information management of the people-money-goods such as health information, financial information, and decision-making information. The $\mathrm{C} / \mathrm{S}$ mode, prototype method, structured system analysis method and object oriented method, etc were used in the system. The SQL Server 2000 database development technology was used as the database [3]. Digital medical treatment is a new type of modern medical 
mode, in which the modern information technology and computer technology are applied to the whole medical process. It is the management goal and development direction of the modern public health. A typical digital medical model is mainly composed of 3 big parts such as the digital medical equipment, hospital network \& management information system, and remote medical service, of which the digital medical and surveillance equipments are the important foundation of digital medical [4]. According to the hospital digitization development experience at home and abroad, the development of the digital hospital can be divided into three stages: the management digital stage, medical digital stage and digital hospital stage featured as the regional medical $[5,6]$. At present, the hospital digital development and construction has entered the third stage in the developed countries such as the United States, Japan and Germany. The construction of digital medical treatment in our country started late. At present, it is in the initial phase of the second stage.

In conclusion, medical digital system developed by the network technology has been the trend of The Times in the rapid development today of the digital technology and network technology.

\section{System Research and Design}

\subsection{Main Function of the System}

The main function of this system is: medical diagnosis, health care, diagnostic tracing and emergency medical treatment, etc. The security, real-time performance, flexibility and intelligent of the system in the development of the system should be considered. The system security includes the security of data transmission, information storage security and the security of the information access. The real-time performance refers to collecting a number of physiological data to users in time by using high precision medical sensors. The data collected will be transmitted to the medical diagnosis and emergency centers in time through a wireless network or mobile network. Medical system will analyze and process the data in time to get a result, which will be feed backed to the users in time. The flexibility can be obtained by using medical sensor with a wireless connection, which is easy to carry and remove. The positioning method can be selected flexibly according to customer's actual environment. The intelligent means that the system can analysis and process the physiological data collected by sensors to judge the optimization treatment scheme, and to provide efficient health services through scheduling the relevant units and personnel in the fastest time for the remote medical care and emergency medical first aid.

\subsection{System Frame Structure}

The frame structure of the system was divided in three levels: the business platform, support platform and foundation platform. (1) The business platform included the user terminal system, security call system, electronic medical records system, hospital information management system, diagnosis expert system and emergency dispatch control system. This layer was the application level to realize the information resource 
digitalization, the business processing networking, management and decision scientization. (2) The support platform provided the system WEB server, data access, transaction processing, security management and other functions. This part provided the supporting environment of the application software system. It provided a variety of services and operational environment for the realization of the function of the application system, and guaranteed the information sharing among systems and among modules. (3) The foundation platform was composed of the mobile and internet platform, database, WEB services, and application services. It provided a support to the system hardware and software. The framework diagram of digital medical system was shown in Figure 1.
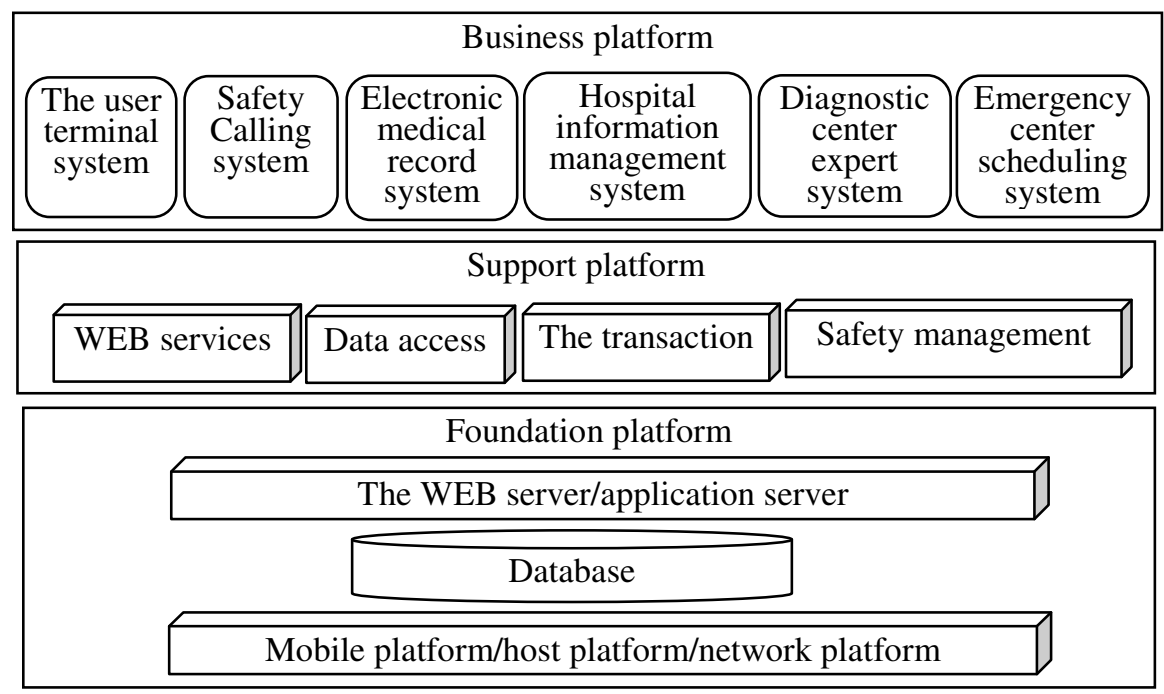

Fig. 1. The framework diagram of digital medical system

\subsection{The Environment of the System Software and Hardware}

The hardware involved in this system was divided into two categories, of which the first was the one about the health care, such as: the pulse sensor, blood pressure sensor, oxygen sensor, temperature sensor, acceleration sensor, electrocardiogram equipment and electroencephalograph, etc. The second type was the one about the computer and network, such as: personal computers, laptops, IPTV, RFID equipment, intelligent mobile phones with the positioning function, PDA, and other equipment.

The input, processing and output flows of an application were separated into the corresponding model layer, view layer and control layer in MVC (model-viewcontrol) design patterns according to the model $\mathrm{M}$, view $\mathrm{V}$ and control $\mathrm{C}$ mode [7]. The application program in the design pattern was divided into the model, view and controller of three different parts, of which each part had its corresponding different functions. 
The application System in community hospitals, medical diagnostic center, and the emergency center were all based on the J2EE platform, but the business logic encapsulated in the EJB containers in the business layer was different. There were J2ME, Windows, Symbian, Mac OS, Android OS, Web OS and OMS platform in the software environment

\subsection{The System Model}

Operations related to a system administrator in the background management terminal of the digital medical system were provided, including the system data update and editing, and the system administrator had the supreme authority over the use of the system. XDS document can be stored in a central database, and can also be stored separately in the local XDS document library of each medical institution. Regional digital medical information system provided the service interface for the client and the regional medical institutions front-end [8]. The regional medical institution frontend was responsible for interfaces of the document extraction, document register, document distribution, collaboration information entering the business system in the business system of medical institutions. Regional information sharing platform centering on resident health records was established, and health data scattered in different institutions were integrated into a complete data information, so that the medical staff can access necessary information timely at any time, any place, in order to provide quality health services [9]. The specific model was shown in Figure 2.

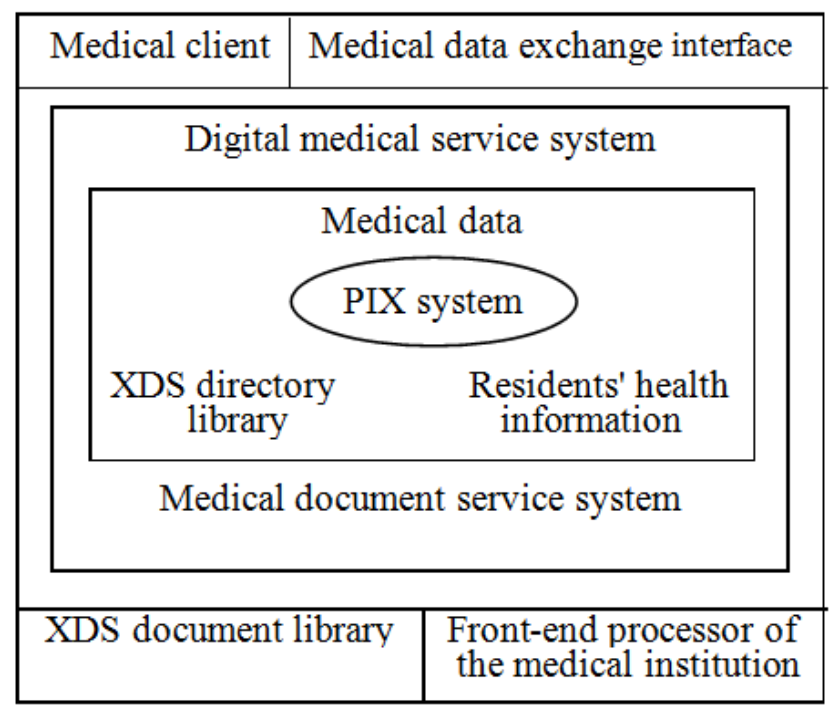

Fig. 2. The specific model of the system 


\subsection{Application System Framework}

An application system framework included the following five layers: client layer, presentation layer, mobile layer, business layer and EIS (enterprise information system) layer.

Client layer: the Web was the main way of J2EE client layer in the system. In addition, there were ways such as the mobile Internet (WAP/HTTP), SMS (CMPP/SGIP), etc.

Presentation layer: the presentation layer mainly included the JSP (Java Server Pages) pages and Java Servlet components of two parts, of which the JSP page programming design realized the view function in the MVC pattern, and the servlet components used in the background service program realized the control function in the MVC pattern.

Mobile layer: this system realized the function of sending and receiving short message via SMS access modules in the mobile layer. Related physiological data, diagnostic results and suggestions, health care information, etc can be sent and received through SMS access modules.

Business layer: EJB was used in the business layer of the system to encapsulate specific business and achieve the model function of the MVC pattern.

EIS (enterprise information system) layer: in this system, ERP and CRM in EIS layer were the existing system of the community hospital, medical diagnostic center, and emergency center.

In order to further improve the effectiveness of medical diagnosis, monitoring control window can be enabled and the current patient physiological information were examined in the current window [10]

\section{System Function Module}

The main function modules of this system included: remote medical diagnosis module, health care module, positioning and tracking module, emergency calls and emergency module.

\subsection{Remote Medical Diagnosis Module Function}

The users needing help can be diagnosed and cured in face to face by doctors and experts in the community hospitals and diagnostic center through IPTV, home computers and mobile terminal. The process was: the user used the dynamic identity to be authentication login and enter the system in the terminal (computer or mobile phone), submitted the consultation application to the hospital diagnosis center, then the application was checked, and the doctor and consultation specific time were arranged after the consultation application was received by the hospital diagnostic center. The hospital diagnosis center will send the consultation information to the applicant or the user by calling system. The confirmation message will be replied by the applicant to the hospital diagnosis center after the consultation arrangement information received. Users were diagnosed in the video by the doctor in the diagnostic center by using the 
video system of a mobile phone or family IPTV. The doctor in the diagnostic center made diagnosis based on user's various physical parameters, electronic medical records and the information obtained through the video. After diagnosis, the doctor filled out the diagnosis report and submitted it to the server. Then, the diagnostic center server sent the diagnosis result to the user. Medical common human physiological parameters were collected through different sensors selected based on different human physiological parameters characteristics [11].

Telemedicine systems can be divided into the acquisition of physiological parameters (completed by physiological parameters collection terminal), data transmission (completed by wireless communication network) and remote monitoring (completed by remote monitoring center server terminal) and other modules, according to their functions and processes [12]. The service system of the regional health information-sharing platform provided service interface for clients of regional health information sharing platform and front-end processor of regional medical institutions [13].

\subsection{Health Care Function Module}

Patients collected the physiological data information with carried medical sensors, and then sent it to the medical diagnosis center. After the medical diagnostic center received the patient's physiological data, the patient's electronic medical record was accessed. Furthermore, both the physiological data information and electronic medical records were sent to the expert system. Based on the patient's electronic medical record information and patient's physiological data, expert system made a scientific judgment, and gave a guideline. If the treatment were needed, it would be sent to the information management module together with the doctor diagnosis information. After the doctor information management module received the diagnosis results from the expert system, the diagnosis and treatment doctor was recommended for the patient, and the recommendation information was sent to the transceiver module. Information transceiver module put forward the advice information to patients. After the treatment information was confirmed by patients, the treatment time and location were confirmed by the doctor information management module, and the detailed treatment information was sent to the medical center. After the medical center received the information, the detailed treatment information was sent to patients. At the same time, the electronic medical records of patients were updated.

\subsection{Positioning and Tracking Module Function}

When users or patients were within the community, the user's accurate location information can be obtained through their carried sensors. This localization method is mainly aimed at children, the elderly and disabled. When patients or monitored person did activities in community, the physiological data information was collected in real time through the carried sensors, and sent it to the diagnosis center. The diagnostic center will send its identity ID to the emergency medical center, based on the monitored person's phone number and the RFID electronic tag. Based on the 
identity ID, the emergency center accessed its electronic medical records and related data in the electronic medical record system, and searched rapidly the right doctors and medical staff. At the same time, the information will be sent to the person's family through the security call system. If necessary, the nearest ambulance and community hospitals will be notified to get the treatment for the patient in the fastest time. The above process requires that the same patient ID be identified with different code in different medical institutions. When the patient referral was happened between different medical institutions, referral or collaborative information need be exchanged to share medical documents. When sharing medical documents, the first is to accurately identify the patient ID, which needs a cross-reference system to link the patient identification code in different medical institutions through the index. When a system needs to be accessed, the patient identification code can be provided in the system [14].

\subsection{Emergency Calls and Emergency Module Function}

When a user or patient had an emergency symptom or sudden emergency, the position information of the person can be obtained in the fastest time through positioning and tracking function. The emergency medical center distributed the ambulance or notified the nearest ambulance to rush to the scene. The medical first aid center accessed the person's electronic medical record information from the electronic medical record system, and sent it to the hospital. At the same time, the injured family information was found, and the person's accident and location information were sent to their relatives through the security call system. The emergency medical center forwarded the person's real-time physiological data and electronic medical records information to the hospital, in order to facilitate making the most effective treatment plan. The emergency medical center determined whether need to inform the police according to the accident type information. If necessary, the nearest police will be informed. The emergency medical center found the injured insurance information, notified the insurance company to is prepared to compensate. Hospitals recorded the relevant medical procedures and information to the person's electronic medical records in the whole course of the treatment. Then, the data in the electronic medical record system was updated.

\section{Discussion and Implication}

The wireless sensing network technology, communication technology, password technology and network technology were integrated in this study system to realize the remote medical treatment, health care, positioning and tracking, emergency calls and first aid, etc. In the paper, details about the system hardware and software platform, system structure and the framework of system function, etc. were illustrated separately in the paper. How to apply the computer technology, network technology and communication technology into the specific examples in the real life of people was elaborated through the general design of digital medical system under the 
network environment. It fully embodies what roles the modern technology can play in today's life. The design of the system is feasible in theory, but in the actual operation and test process, there may be a few problems needed to be solved. It will be continuously developed and improved in the future.

\section{References}

1. Egan, G.F., Liu, I.Q.: Computers and Networks in Medical and Healthcare Systems. Computers in Biology and Medicine 25, 355-365 (1995)

2. Li, G.: Design and implementation of mobile medical emergency self-help system based on the 3 G. Shanghai (2009)

3. Hua, X.: Design and development of hospital information management system. Chengdu (2012)

4. Cheng, N.: Digital medical. Digital life 3, 33 (2001)

5. Li, H.: Digital hospital—-hospital mode in the future. Medical and Health Care Equipment 12, 126-127 (2003)

6. Li, L.: Digital hospital— The necessity of the hospital modernization developing. China Journal of Modern Medicine 21, 153-155 (2004)

7. John, D.: Model-View-Cont roller (MVC) Architeture (2000), http: / /www.jdl.co.uk/bridfings /MVC.pdf

8. Bai, J.: Intelligent Community Health China medical device information 2, 14-16 (2000)

9. Liu, G.: The Design and Development of the Transmission Platform of Community Telemedicine System based on Web. Tianjin Medical University (May 2009)

10. Wu, Q.: Remote Medical Monitoring System based on Wireless Sensor Networks. University of Electronic Science and Technology (February 2006)

11. Yang, Y., Wang, L.: Architecture for body sensor networks. In: IEEE Proceedings of the Perspective in Pervasive Computing, vol. 03, pp. 23-28 (2005)

12. Zhao, Z., Cui, L.: A Remote Health Care System Based on Wireless Sensor Networks. Information and Control (2), 265-269 (2006)

13. Bai, J.: Intelligent Community Health Care System. China Medical Devices Information (2), 14-16 (2000)

14. Li, Z., Gong, X., Yuan, C.: The Development Status and Problems of Community Health Service in China. Chinese Primary Health Care (11), 31-32 (2007) 